\title{
Mapping the stripe rust resistance gene in Chinese wheat Guinong 775
}

\author{
Luhua $\mathrm{Li}^{\mathrm{a}}{ }^{\mathrm{b}}$, Mingjian Ren ${ }^{\mathrm{a}, \mathrm{b}}$, Fang $\mathrm{He}^{\mathrm{a}, \mathrm{b}}$, Ruhong $\mathrm{Xu}^{\mathrm{a}, \mathrm{b}, *}$ \\ a College of Agriculture, Guizhou University, Guiyang 550025, China \\ b Guizhou Subcentre of National Wheat Improvement Centre, Guiyang 550025, China
}

*Corresponding author, e-mail: xrhgz@163.com

Received 26 Dec 2018

Accepted 19 Jun 2019

\begin{abstract}
Wheat stripe rust, caused by Puccinia striiformis Eriks. f. sp. tritici, is a destructive wheat disease worldwide. To find new sources of resistance, Guinong 775 wheat was previously bred and identified as having a strong resistance against stripe rust, powdery mild and leaf rust. Guinong 775 showed a strong resistance against mixed fungi (CYR32 and CH42) associated with stripe rust that are common in Guiyang and Qianxinan. It was crossed with the susceptible wheat line Huixianhong to develop F1, F2 and F3 hybrids. In this study, 173 simple sequence repeat markers were used to map the stripe rust resistance gene in Guinong 775, and the resistance phenotype of Guinong 775 was regulated by a single dominant gene named YrGn775. YrGn775 was mapped to chromosome 1BS with the marker Xgwm413 flanking the gene at a distance of $12.0 \mathrm{cM}$. Genetic and molecular marker analyses demonstrated that this dominant gene may have originated in Aegilops ventricosa. Thus YrGn775 may be a novel gene in wheat.
\end{abstract}

KEYWORDS: stripe rust,simple sequence repeat, single dominant gene, Xgwm413

\section{INTRODUCTION}

Stripe or yellow rust (YR), caused by Puccinia striiformis Eriks. f. sp. tritici, is a destructive disease of common wheat and is a constant threat to wheat production $(5-25 \% \text { yield losses })^{1}$. The development of resistant commercial cultivars with effective YR resistance $(\mathrm{Yr})$ genes is the most effective, economical and environmentally friendly strategy to control stripe rust ${ }^{2}$. At present, $130 \mathrm{Yr}$ resistance genes have been documented, among which 83 have been formally designated and 47 have been temporarily designated ${ }^{3}$. Although the number of documented $\mathrm{Yr}$ resistance genes is increasing, new variants can be generated in $P$. striiformis ${ }^{4}$, resulting in most stripe rust resistant wheat varieties losing their resistance. Hence identifying new durable resistance genes to stripe rust has been emphasized in international wheat breeding.

Molecular markers, such as sequencecharacterized amplified regions ${ }^{5}$, restriction fragment length polymorphisms ${ }^{6}$ and simple sequence repeats $(\mathrm{SSRs})^{7}$, have been widely used for mapping stripe rust resistance genes in wheat. However, owing to the large allohexaploid genome $(17 \mathrm{~Gb})$ of wheat, the fine mapping of resistance genes is difficult and time-consuming ${ }^{8}$. Currently, quantitative trait locus (QTL) mapping is the preferred method of mapping wheat resistance genes. QTL mapping requires that entire populations be analysed, and the lack of highdensity polymorphic markers limits the application of QTL mapping ${ }^{9}$. Bulked segregant analyses (BSAs) involving selected and pooled DNA samples from two contrasting groups, such as biparental populations of individuals, have been modified by using large populations, high-density markers and increased tail sizes to map the target genes. Compared with QTL mapping, a BSA significantly reduces the scale and cost by simplifying the mapping procedure ${ }^{10}$.

Aegilops species are important genetic sources for improving the variability of cultivated wheat, and formally designated resistance genes for stripe rust, such as $\mathrm{Yr} 28, \operatorname{Yr} 17$ and $\mathrm{YrCH} 42$, have been transferred to wheat from various Aegilops species ${ }^{11}$. Martynov et al demonstrated that the percentage of wheat varieties containing Aegilops genetic materials has gradually increased and that they have retained their effectiveness. From 19622011, wheat varieties created with the involvement of Aegilops tauschii increased from $0 \%$ to $14-18 \%$, while those with Aegilops ventricosa increased from $1 \%$ to $34-37 \%{ }^{12}$. Meanwhile, others powdery mildew and leaf rust resistant genes have also trans- 
ferred to wheat from various Aegilops species.

Wheat germplasm Guinong 775 is a cultivar obtained in $1995^{13}$. Because Guinong 775 was identified as being resistant to stripe rust and powdery mildew by the Chinese Academy of Sciences of Beijing and Northwest A\&F University of Shanxi in China, it has been widely used as a parent in wheat breeding in the Guizhou Province of China. In addition, Guinong 775 has provided strong resistance against stripe rust and powdery mildew according to several years of field observations. However, the genetic basis of cultivar Guinong 775's stripe rust resistance is not clear. Here, we explore the genomic locations of the potential stripe rust resistance genes of Guinong 775.

\section{MATERIALS AND METHODS}

\section{Plant materials}

Guinong 775, as a wheat material immune to stripe rust and powdery mildew, is cultivated from complex wide-crossing hybrids of A. tauschii Coss/Avena fatua Linn/A. ventricosa Tausch/Sauwne20. Guinong 775 has been stored in the Wheat Research Center at the College of Agriculture in Guizhou University since $1995^{13}$. Wheat line Huixianhong, with a high susceptible to stripe rust and powdery mildew was provided by Northwest A\&F University.

\section{Resistance test for stripe rust in the field}

Guinong 775, Huixianhong, and the $\mathrm{F}_{2: 3}$ populations from Guinong $775 \times$ Huixianhong were tested for the presence of stripe rust resistance genes, in the field in Huaxi, Guiyang and at the Institute of Agricultural Science of Qianxinan. Equal amounts of $P$. striiformis pathotypes CYR32 and CH42, which are common in Guiyang and Qianxinan, respectively, were mixed and sprayed onto rows at tilling. When the disease severity reached 90-100\%, then the infection types (ITs) were investigated. The ITs were scored on a $0-4$ scale according to Roelfs ${ }^{15}$ : 0 indicated immune, no lesion in plant; 0 : indicated nearly immune, hypersensitive reaction and dead spots in leaf; 1 indicated highly resistant, the diameter of lesion is less than $1 \mathrm{~mm}$, sparse mycelium, occasionally larger lesions but still green and rare sporulation; 2 indicated mildly resistant, the diameter of lesion is less than $1 \mathrm{~mm}$, thicker mycelium, green-proof and can produce a certain amount of spores; 3 indicated mildly susceptible, leaf lesions are numerous, the diameter of lesion is greater than $1 \mathrm{~mm}$, thick mycelium, larger spores but the lesion is not patchy; and 4 indicated highly susceptible, the diameter of lesion is greater than $1 \mathrm{~mm}$, thick mycelium, larger spores and form lesion patches.

\section{DNA extraction}

Fresh leaf tissues of five-leaf stage plants were cut and stored at $-80^{\circ} \mathrm{C}$. Then the CTAB method was used to carry out DNA extraction ${ }^{14}$.

\section{PCR analysis}

PCR amplification was performed in $20 \mu \mathrm{l}$ reaction volumes containing $10 \mathrm{mM}$ Tris- $\mathrm{HCl}(\mathrm{pH} 8.8)$, $50 \mathrm{mM} \mathrm{KCl}, 2.0 \mathrm{mM} \mathrm{MgCl}, 0.08 \%$ Nonidet P40, $0.2 \mathrm{mM}$ dNTPs, 1 unit Taq DNA polymerase, $0.4 \mu \mathrm{M}$ of each primer (Sangon Biotech, China) and 35$50 \mathrm{ng}$ of genomic DNA. Specific primer amplification was performed with following program: $5 \mathrm{~min}$ at $94^{\circ} \mathrm{C}$ (pre-denaturation), followed by 35 cycles of $45 \mathrm{~s}$ at $94^{\circ} \mathrm{C}, 45 \mathrm{~s}$ at $50-61^{\circ} \mathrm{C}$ (according to the specific primer) and $1 \mathrm{~min}$ at $72^{\circ} \mathrm{C}$, then by a final extension of $10 \mathrm{~min}$ at $72{ }^{\circ} \mathrm{C}$. The PCR products were detected on a $5 \%$ non-denatured PAGE combined with silver staining ${ }^{14}$. Gels were then visualized.

\section{Inheritance analysis and linkage map construction of resistance genes}

The segregation of the resistance genes in the $F_{2: 3}$ populations were detected by $\chi^{2}$-tests. Specific primers were used to amplify and classify the genotypes of the populations. A and B indicated that the band type of the molecular marker was the same as in Guinong 775 and Huixianhong, respectively. $\mathrm{H}$ indicated the heterozygous lines and - indicated a missing or not detected sample. MAPMAKER 3.0 was used to carry out molecular marker and resistance linkage analyses, and the linkage map was constructed using MAP DRAW V2.1.

\section{RESULTS}

\section{Parental ITs and the inheritance analysis}

Wheat seedlings were infected with mixed fungi, isolates CYR32 and CH42, associated with stripe rust that are common in Guiyang and Qianxinan, respectively. Guinong 775 had durable immune resistance $(\mathrm{IT}=0)$ to stripe rust for over 20 years of field testing, and the wheat line Huixianhong was susceptible $(\mathrm{IT}=4)$ to stripe rust. An $\mathrm{F}_{1}$ population of the cross Guinong $775 \times$ Huixianhong exhibited immune resistance (IT $=0$ ) to stripe rust, whereas the $F_{2: 3}$ population of 131 lines in Guiyang and 63 lines in Qianxinan exhibited segregated stripe 
Table 1 Stripe rust infection levels of parents, and an inheritance analysis of the $\mathrm{F}_{2: 3}$ population of the Guinong $775 \times$ Huixianhong cross!

\begin{tabular}{|c|c|c|c|c|c|c|c|c|c|}
\hline \multirow[t]{2}{*}{ Population } & \multirow[t]{2}{*}{ Progeny } & \multicolumn{6}{|c|}{ Number of seedlings } & \multirow{2}{*}{$\begin{array}{c}\text { Expected } \\
\text { ratio }\end{array}$} & \multirow[t]{2}{*}{$\chi^{2}$} \\
\hline & & 0 & $0:$ & 1 & 2 & 3 & 4 & & \\
\hline Guinong 775 & $\mathrm{P} 1$ & 50 & & & & & & & \\
\hline Huixianhong & $\mathrm{P} 2$ & & & & & & 50 & & \\
\hline Guinong $775 \times$ Huixianhong & $\mathrm{F}_{1}$ & 12 & & & & & & & \\
\hline Guinong $775 \times$ Huixianhong & $\mathrm{F}_{2: 3}$ & 68 & 37 & 27 & 15 & 16 & 31 & $3: 1$ & 0.0275 \\
\hline
\end{tabular}

${ }^{\dagger}$ Plants belong to ITs 0-2 scale were grouped into resistant and 3-4 scale were grouped into susceptible for testing the 3:1 ratio. $\chi^{2}$ represents the chi squared value of 3:1, $\chi_{0.05}^{2}=3.841$. The calculated $\chi^{2}<\chi_{0.05}^{2}$ demonstrated a significant difference of the value.

rust resistance. Among the 194 lines in Guiyang and Qianxinan, 68 lines were immune resistant $(\mathrm{IT}=0), 37$ lines were nearly immune resistant (IT =0:), 27 lines were highly resistant (IT $=1$ ), 15 lines were mildly resistant (IT $=2$ ), 16 lines were mildly susceptible (IT $=3$ ), and 31 lines were susceptible (IT $=4$ ) (Table 1$)$. The $\chi^{2}$ results of the combined $\mathrm{F}_{2: 3}$ population lines $\left(\chi_{3: 1}^{2}=0.028<\right.$ $\left.\chi_{0.05}^{2}=3.841\right)$ demonstrated that one independent dominant complementary gene participated in the stripe rust resistance of Guinong 775 (Table 1). The gene was named YrGn775.

\section{Molecular markers screening}

In total, 173 pairs of SSR primers, including those specifically linked to stripe rust resistance genes, were used for screening the resistance gene of Guinong 775. We found 52 pairs of SSR primers displayed polymorphisms in the parents. Additionally, highly resistant and susceptible pools were constructed according to the BSA using genomic DNA mixtures of 10 homozygous $\mathrm{F}_{2: 3}$ lines each. Further, among the 52 pairs of SSR primers we found Xgwm413, Xbarc187, Xgwm131, Xgwm169 and $X b a r c 3$ displayed polymorphisms in the resistant and susceptible pools, and the primer sequence are listed (Table 2). These five dominant markers were then used to analyse two different collection sites, the 131 lines in Guiyang and the 63 lines in Qianxinan that constituted the $\mathrm{F}_{2: 3}$ population. Only Xgwm413 was determined to be linked with YrGn775 (Fig. 1, Table 3).

\section{Genomic location of $\mathrm{YrGn} 775$}

Based on the GrainGenes2.0 database, Xgwm413 was located in chromosome 1BS. Among the 147 stripe rust resistant $\mathrm{F}_{2: 3}$ lines identified with the Xgwm413 primer, 52 lines amplified the same band as Guinong 775, 14 lines amplified the same band as Huixianhong, and 81 lines were heterozygous (Fig. 1a, Table 3). Meanwhile, among the 47 stripe rust susceptible $\mathrm{F}_{2: 3}$ lines, 3 lines amplified the same band as Guinong 775, 39 lines amplified the same band as Huixianhong and 5 lines were heterozygous (Fig. 1b). The $\mathrm{F}_{2: 3}$ population results (Fig. 1, Table 3) demonstrated that the dominant YrGn775 gene was located in chromosome 1BS, which was $12.0 \mathrm{cM}$ from marker Xgwm413 (Fig. 2).

\section{Source of $\mathrm{YrGn} 775$}

The Xgwm413 marker was used to identify the pedigree parents of YrGn775. The Xgwm413 primer could amplify a specific band in Guinong 775 and A. ventricosa Tausch. However, there was no specific band present in A. tauschii Coss, A. fatua Linn, Sauwne20 or Haynaldia villosa (Fig. 3a). Hence YrGn775 may originate from $A$. ventricosa Tausch. The Xgwm413 and Xgwm11 markers were linked to the stripe rust resistant gene YrH52 in Triticum dicoccoides at distances of $1.3 \mathrm{cM}$ and $2.3 \mathrm{cM}$, respectively ${ }^{16}$. Then these two markers were used to detect Guinong 775, A. ventricosa and T. dicoccoides to verify if $\mathrm{YrGn775}$ is YrH52. We found the Xgwm413 and Xgwm11 markers could amplify a specific band in Guinong 775 and A. ventricosa Tausch (Fig. 3b), which differed from the band in T. dicoccoides. The results indicated that $\operatorname{YrGn775}$ was not YrH52 and further confirmed that $\mathrm{YrGn} 775$ originated from $A$. ventricosa Tausch.

\section{DISCUSSION}

Most wheat cultivars have gradually lost their resistance to the fungi isolates CYR32 and especially CH42 in China. Han et al $^{17}$ demonstrated that the resistance of Guinong 775 was conferred by two different single dominant genes to CYR32 and $\mathrm{CH} 42$. An allelic analysis indicated that the gene providing resistance to CYR32 was linked to the 
Table 2 Sequences of five specific markers for the bulked segregant analysis.

\begin{tabular}{llcc}
\hline Marker & Primer sequence $\left(5^{\prime}-3^{\prime}\right)$ & Location & Tm \\
\hline Xgwm413 & TGCTTGTCTAGATTGCTTGGG GATCGTCTCGTCCTTGGCA & $1 \mathrm{~B}$ & $58^{\circ} \mathrm{C}$ \\
Xbarc187 & GTGGTATTTCAGGTGGAGTTGTTTA CGGAGGAGCAGTAAGGAAGG & $1 \mathrm{~B}$ & $58^{\circ} \mathrm{C}$ \\
Xgwm131 & AATCCCCACCGATTCTTCTC AGTTCGTGGGTCTCTGATGG & $1 \mathrm{~B}$ & $60^{\circ} \mathrm{C}$ \\
Xgwm169 & ACCACTGCAGAGAACACATACG GTGCTCTGCTCTAAGTGTGGG & $6 \mathrm{~A}$ & $60^{\circ} \mathrm{C}$ \\
Xbarc3 & TTCCCTGTGTCTTTCTAATTTTTTTT GCGAACTCCCGAACATTTTTAT & $6 \mathrm{~A}$ & $52^{\circ} \mathrm{C}$ \\
\hline
\end{tabular}

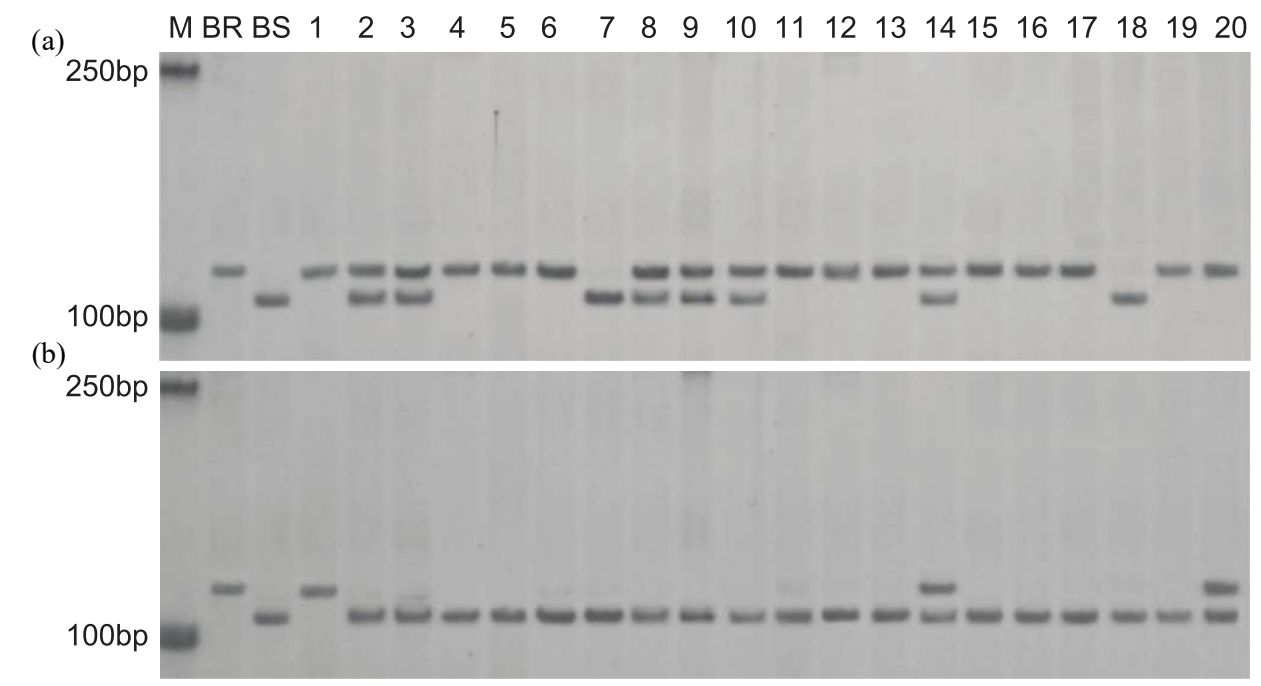

Fig. 1 Marker analysis of the $\mathrm{F}_{2: 3}$ population. (a) Detection results of Xgwm413 primers in the resistant lines. (b) Detection results of Xgwm413 primers in the susceptible lines. M: 100-bp marker; BR: stripe rust resistant pool; BS: stripe rust susceptible pool; 1-20: $\mathrm{F}_{2: 3}$ lines.

Table 3 Band types of Xgwm413 marker in $\mathrm{F}_{2: 3}$ lines!

\begin{tabular}{|c|c|c|c|c|c|c|c|}
\hline \multirow[t]{2}{*}{ Marker } & \multicolumn{3}{|c|}{1} & \multicolumn{3}{|c|}{4} & \multirow[t]{2}{*}{$\chi_{1: 2: 1}^{2}$} \\
\hline & A & $\mathrm{H}$ & B & A & $\mathrm{H}$ & B & \\
\hline Xgwm413 & 52 & 81 & 14 & 3 & 5 & 39 & 0.440 \\
\hline \multicolumn{8}{|c|}{$\begin{array}{l}1 \text {, the } 147 \text { stripe rust resistant } \mathrm{F}_{2: 3} \text { lines }(\mathrm{IT}=0-2) ; 4 \text {, } \\
\left.\text { the } 47 \text { stripe rust susceptible } \mathrm{F}_{2: 3} \text { lines (IT }=3-4\right) ; \mathrm{A} \text {, } \\
\text { the band type of the molecular marker was the same as } \\
\text { that of Guinong } 775 ; \mathrm{B} \text {, the band type of the molecular } \\
\text { marker was the same as that of Huixianhong; } \mathrm{H} \text {, the } \\
\text { heterozygous lines. }\end{array}$} \\
\hline
\end{tabular}

formally designated stripe rust resistant genes $\mathrm{Yr} 24$ and $\mathrm{Yr} 26$, and that the gene providing resistance to CH42 may be a novel gene ${ }^{17}$. However, the CH42-associated resistance gene of Guinong 775 has not been mapped. In this study, we identified a single dominant gene from the Chinese wheat line Guinong 775 that shows a high resistance to mixed fungi (isolates CYR32 and CH42) associated with stripe rust that are common in Guiyang and Qianxinan, Guizhou Province, China (Table 1).

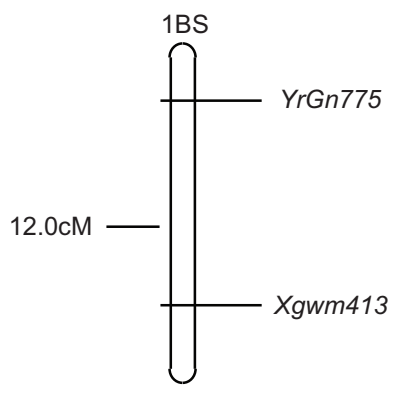

Fig. 2 Genetic map of $Y r G n 775$.

Here, we mapped the dominant gene from a cross between Guinong 775 and Huixianhong. Only one specific marker, Xgwm413, was selected from 173 SSR primer pairs to map the resistance gene, named YrGn775.

The Xgwm413 marker is located in chromosome 1BS according to the GrainGenes2.0 database. At present, there are nine stripe rust resistance genes documented in chromosome 1BS, among which six (Yr10, Yr15, Yr24, Yr26, Yr64 and 

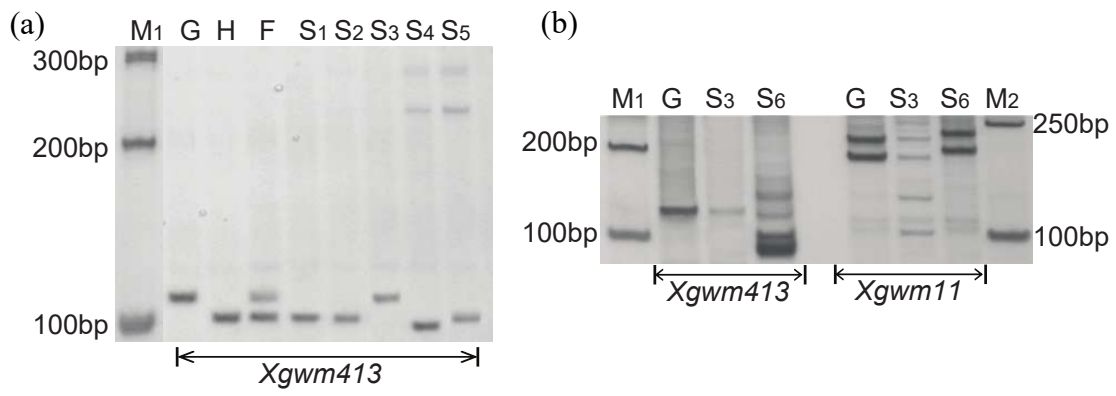

Fig. 3 The source detection of $Y r G n 775$. (a) The Xgwm413 primer amplification results in the pedigree parents. (b) The Xgwm413 and Xgwm11 primer amplification results in S3 and S6. $\mathrm{M}_{1}$ : 100-bp marker; G: Guinong 775; $\mathrm{H}$ : Huixianhong; $\mathrm{F}$ : the $\mathrm{F}_{1}$ line of Guinong $775 \times$ Huixianhong cross; S1: A. tauschii Coss; S2: A. fatua Linn; S3: A. ventricosa Tausch; S4: Sauwne20; S5: H. villosa; S6: T. dicoccoides; M2: DL2000 marker.

Yr65) have been formally designated and three (YrAlp, $\mathrm{YrC1} \mathrm{H2}$ and $\mathrm{YrH52}$ ) have been temporarily designated ${ }^{3}$. SSR markers Xpsp3000, Xgwm33, Xbarc187, Xgwm11/Xgwm18, Xgdm33, Xgwm18, Xgwm18, Xgwm273 and Xgwm413 are closely linked to Yr10, Yr15, Yr24, Yr26, Yr64, Yr65, YrAlp, YrC142 and YrH52, respectively. All of these SSR markers were used to screen for the resistance gene of Guinong 775. The Xbarc187 and Xgwm413 markers displayed polymorphisms in the parents (Table 2). The $\mathrm{F}_{2: 3}$ population analysis indicated that only Xgwm413 was linked to $\mathrm{YrGn} 775$ (Fig. 1, Table 3). The specific markers $X$ barc187 and Xgwm11/Xgwm18 that are closely linked to $\mathrm{Yr} 24$ and $Y r 26$, respectively, were not linked to $Y r G n 775$, which indicated that $Y r G n 775$ may be the stripe rust resistance gene to isolates of CH42 in Guinong 775.

The Xgwm413 marker detection results indicated that $\mathrm{YrGn} 775$ was in chromosome 1BS (Fig. 1) and $12.0 \mathrm{cM}$ from the $\mathrm{YrGn} 775$ gene (Fig. 2). Aegilops species are sources of stripe rust resistance genes, and partly resistant Aegilops genes have been introgressed into common wheat for the cultivation of cultivars with resistance to stripe rust ${ }^{11}$. The wheat Guinong 775 was obtained from complex wide-crossing hybrids of A. tauschii Coss/A. fatua Linn/A. ventricosa Tausch/Sauwne20. Also, Guinong775 had been proved possess $6 \mathrm{VS} / 6 \mathrm{AL}$ translocation chromosome and the powdery mildew resistance is correlative to the alien chromosome arm, original from $\mathrm{H}$. vil$\operatorname{los}^{18}{ }^{18}$. The pedigree parents of $\mathrm{YrGn775}$ were identified using the Xgwm413 marker (Fig. 3a), and the result indicated that the specific resistance gene may have originated from $A$. ventricosa Tausch. Peng et al reported that the Xgwm413 marker is linked with $\mathrm{YrH} 52$ in T. dicoccoides at $1.3 \mathrm{cM}^{19}$. Hence the resistance gene $\mathrm{YrGn775}$ could be $\mathrm{YrH52}$ or may be a new stripe rust resistance gene. Further, we detected the Xgwm413 and Xgwm11 markers which were linked to YrH52 in T. dicoccoides. The detection result of Xgwm413 marker showed that Guinong 775 and A. ventricosa Tausch amplified the same specific band, however T. dicoccoides did not, which demonstrated that $\operatorname{YrGn775}$ may originate from A. ventricosa Tausch and was not YrH52 (Fig. 3b left). In addition, the Xgwm11 marker test coincided with the result of Xgwm413 marker (Fig. 3b right) further indicated that $Y r G n 775$ originated from $A$. ventricosa Tausch and that it may be a novel resistant gene of stripe rust in wheat.

Stripe rust has a long history, wide distribution and rapid ability to spread, making it a devastating wheat disease. Because P. striiformis is highly variable and evolves new virulent races, resistance genes gradually lose their effectiveness and the corresponding wheat cultivars lose their resistance ${ }^{20}$. Hence wheat breeders need to identify new resistance genes to improve the spectrum and persistence of stripe rust resistance. We observed that the wheat line Guinong 775 exhibited a persistent resistance for over 20 years to stripe rust, which is common in Guizhou Province. Our results confirmed that the resistance of Guinong 775 was controlled by a single dominant gene, consistent with the results of Han et al ${ }^{15}$. Here, the resistance gene $\operatorname{YrGn775}$ was mapped to chromosome 1BS. The genetic distance of $12.0 \mathrm{cM}$ from Xgwm413 to $\mathrm{YrGn} 775$ is still large; hence the genetic populations studied will be increased and more molecular markers will be explored to confirm the specific location of $Y r G n 775$.

In summary, this study confirmed that the persistent stripe rust resistance to $P$. striiformis in 
Guinong 775 wheat is controlled by a single dominant gene. The genomic location analysis demonstrated that $Y r G n 775$ localized in chromosome 1BS and may be a novel stripe rust resistance gene.

Acknowledgements: This project was supported by grants from the National Key Research Project (Seven Major Crop Breeding), Key Project of China (2017YFD0100900), the National Natural Science Foundation of China (31660390), the Agriculture Achievements Transformation Project in Guizhou ([2016] 4022), Construction Program of Biology First-class Discipline in Guizhou (GNYL [2017] 009), the Guizhou Science and Technology Plan Project ([2019] 1073), and the Introducing Talents Research Project of Guizhou University ([2017] 49). We thank Prof Zhensheng Kang of Northwest A\&F University for kindly provide wheat line Huixianhong. And we also thank International Science Editing for editing this manuscript.

\section{REFERENCES}

1. Wellings CR (2011) Global status of stripe rust: a review of historical and current threats. Euphytica 179, 129-141.

2. Maccaferri M, Zhang J, Bulli P, Abate Z, Chao S, Cantu D, Bossolini E, Chen X, et al (2015) A genome-wide association study of resistance to stripe rust (Puccinia striiformis $\mathrm{f}$. sp. tritici) in a worldwide collection of hexaploid spring wheat (Triticum aestivum L.). G3 (Bethesda) 5, 449-465.

3. McIntosh RA, Dubcovsky J, Rogers W, Morris C, Appels R, Xia XC (2016) Catalogue of gene symbols for wheat: 2015-2016 supplement. Available at: https://shigen.nig.ac.jp/wheat/komugi/genes/ macgene/supplement2015.pdf.

4. Zheng L, Lu X, Liang X, Jiang S, Zhao J, Zhan G, Liu P, Wu J, et al (2017) Molecular characterization of novel totivirus-like double-stranded RNAs from Puccinia striiformis f. sp. tritici, the causal agent of wheat stripe rust. Front Microbiol 8, 1960.

5. Abdollahi Mandoulakani B, Yaniv E, Kalendar R, Raats D, Bariana HS, Bihamta MR, Schulman AH (2015) Development of IRAP- and REMAP-derived SCAR markers for marker-assisted selection of the stripe rust resistance gene Yr15 derived from wild emmer wheat. Theor Appl Genet 128, 211-219.

6. Dawson AM, Ferguson JN, Gardiner M, Green P, Hubbard A, Moscou MJ (2016) Isolation and fine mapping of Rps6: an intermediate host resistance gene in barley to wheat stripe rust. Theor Appl Genet 129, 831-843.

7. Wu J, Zeng Q, Wang Q, Liu S, Yu S, Mu J, Huang S, Sela H, et al (2018) SNP based pool genotyping and haplotype analysis accelerate fne mapping of the wheat genomic region containing stripe rust resistance gene Yr26. Theor Appl Genet 131, 1481-1496.

8. Wang Y, Xie J, Zhang H, Guo B, Ning S, Chen Y, Lu P, Wu Q, et al (2017) Mapping stripe rust resistance gene $\mathrm{YrZH} 22$ in Chinese wheat cultivar Zhoumai 22 by bulked segregant RNA Seq (BSR Seq) and comparative genomics analyses. Theor Appl Genet 130, 2191-2201.

9. Yang H, Li C, Lam HM, Clements J, Yan G, Zhao SC (2015) Sequencing consolidates molecular markers with plant breeding practice. Theor Appl Genet 128, 779-795.

10. Zou C, Wang P, Xu Y (2016) Bulked sample analysis in genetics, genomics and crop improvement. Plant Biotechnol $J$ 14, 1941-1955.

11. Toor PI, Kaur S, Bansal M, Yadav B, Chhuneja P (2016) Mapping of stripe rust resistance gene in an Aegilops caudata introgression line in wheat and its genetic association with leaf rust resistance. $J$ Genet 95, 933-938.

12. Martynov SP, Dobrotvorskaya TV, Mitrofanova OP (2015) Genealogical analysis of the use of Aegilops (Aegilops L.) genetic material in wheat (Triticum aestivum L.). Russ J Genet 51, 855-862.

13. Zhang Q (1999) The mothodology of multiresistance to diseases in wheat distant breeding. Southwest China J Agric Sci 12, 32-38.

14. Wan J, Ren M, Chen T, Xu R (2011) Molecular detection of stripe rust resistant genes in 108 wheat germplasms. Guizhou Agric Sci 39, 22-26.

15. Roelfs AP (1984) Race specificity and methods of study. In: Roelfs AP, Bushnell WR (eds), The Cereal Rusts: Origins, Specificity, Structure, and Physiology, Academic Press, Orlando, pp 131-164.

16. Robert O, Abelard C, Dedryver F (1999) Identification of molecular markers for the detection of the yellow rust resistance gene $\mathrm{Yr} 17$ in wheat. $\mathrm{Mol}$ Breeding 5, 167-175.

17. Han DJ, Wang N, Jiang Z, Wang QL, Wang XJ, Kang ZS (2012) Characterization and inheritance of resistance to stripe rust in the wheat line Guinong 775. Yi Chuan 34, 1607-1613.

18. Yuan H, Li Q, Dai X (2009) Cytology analysis of resistant wheat germplasm Guinong 775. J Southwest China Normal Univ Natur Sci 34, 137-141.

19. Peng JH, Fahima T, Röder MS, Li YC, Dahan A, Grama A, Ronin YI, Korol AB, et al (1999) Microsatellite tagging of the stripe rust resistance gene YrH52 derived from wild emmer wheat, Triticum dicoccoides, and suggestive negative crossover interference on chromosome 1B. Theor Appl Genet 98, 862-872.

20. Zhan GM, Chen XM, Kang ZS, Huang LL, Wang MN, Wan AM, Cheng P, Cao SQ, et al (2012) Virulence and molecular comparison of Puccinia striiformis $\mathrm{f}$. sp. tritici populations in China and the United States. Fungal Biol 116, 643-653. 